Research Article

\title{
Relationship between Driving Pressure and Mortality in Ventilated Patients with Heart Failure: A Cohort Study
}

\author{
Qilin Yang $(\mathbb{D}$, Jiezhao Zheng $(\mathbb{D}$, Xiaohua Chen, Weiyan Chen, Deliang Wen, \\ Xuming Xiong $\mathbb{B}$, and Zhenhui Zhang \\ Department of Critical Care, The Second Affiliated Hospital of Guangzhou Medical University, No. 250 Changgang East Road, \\ Haizhu District, Guangzhou, China
}

Correspondence should be addressed to Xuming Xiong; xiongxuming9@126.com and Zhenhui Zhang; zhzhhicu@126.com

Received 21 January 2021; Revised 9 October 2021; Accepted 17 November 2021; Published 29 November 2021

Academic Editor: Paola Pierucci

Copyright (c) 2021 Qilin Yang et al. This is an open access article distributed under the Creative Commons Attribution License, which permits unrestricted use, distribution, and reproduction in any medium, provided the original work is properly cited.

Background. Heart failure (HF) is a leading cause of mortality and morbidity worldwide, with an increasing incidence. Invasive ventilation is considered to be essential for patients with HF. Previous studies have shown that driving pressure is associated with mortality in acute respiratory distress syndrome (ARDS). However, the relationship between driving pressure and mortality has not yet been examined in ventilated patients with HF. We assessed the association of driving pressure and mortality in patients with HF. Methods. We conducted a retrospective cohort study of invasive ventilated adult patients with HF from the Medical Information Mart for Intensive Care-III database. We used multivariable logistic regression models, a generalized additive model, and a two-piecewise linear regression model to show the effect of the average driving pressure within $24 \mathrm{~h}$ of intensive care unit admission on in-hospital mortality. Results. Six hundred and thirty-two invasive ventilated patients with HF were enrolled. Driving pressure was independently associated with in-hospital mortality (odds ratio [OR], 1.12; 95\% confidence interval [CI], $1.06-1.18 ; P<0.001)$ after adjusted potential confounders. A nonlinear relationship was found between driving pressure and inhospital mortality, which had a threshold around $14.27 \mathrm{cmH}_{2} \mathrm{O}$. The effect sizes and CIs below and above the threshold were 0.89 (0.75 to 1.05 ) and 1.17 (1.07 to 1.30), respectively. Conclusions. There was a nonlinear relationship between driving pressure and mortality in patients with HF who were ventilated for more than $48 \mathrm{~h}$, and this relationship was associated with increased inhospital mortality when the driving pressure was more than $14.27 \mathrm{cmH}_{2} \mathrm{O}$.

\section{Introduction}

Heart failure (HF) is a syndrome caused by structural or functional cardiac abnormalities that lead to elevated intracardiac pressures or a reduced cardiac output. HF is a leading cause of mortality and morbidity worldwide [1-3]. Previous studies have shown that the prevalence of HF increased by as much as 30\% in Medicare beneficiaries from 1994 to 2003 [4]. In the study of Atherosclerosis Risk in Communities (ARIC) of the National Heart, Lung, and Blood Institute (NHLBI), the 30-day, 1-year, and 5-year case fatality rates after hospitalization for HF were up to $10.4 \%$, $22 \%$, and $42.3 \%$, respectively [5]. HF is commonly complicated by pulmonary edema. Approximately half of patients with acute respiratory failure with cardiogenic shock and acute myocardial infarction require ventilation, especially invasive mechanical ventilation [5-8].

Driving pressure (plateau pressure (Pplat) - positive endexpiratory pressure (PEEP)) has recently received attention and has been widely studied as a static index indicating the inflation characteristics of pulmonary alveoli during the individual respiratory cycle $[9,10]$. The lung elastance is computed as the ratio between driving pressure and tidal volume $(V t)$, so the driving pressure can also be calculated as $V t *$ respiratory system elastance. Therefore, by assuming that the tidal volume is fixed during mechanical ventilation, it is clear to understand how the driving pressure is strictly correlated with the mechanical features of the respiratory system. Moreover, ventilated patients occasionally present with ventilator-induced lung injury (VILI), which increases mortality [11]. Some studies 
have suggested that VILI is partly caused by respiratory system elastance and have shown that driving pressure is a good predictor of VILI $[12,13]$.

The relationship between driving pressure and outcomes has gained increasing interest, especially in acute respiratory distress syndrome (ARDS) [14, 15]. Relevant studies have shown that lower driving pressure could decrease lung overstress and was associated with lower mortality in patients with ARDS [14-17]. However, the increased risk of poor prognosis associated with high drive pressure has been observed not only in patients with ARDS but also in ventilated patients, which includes patients with severe pneumonia without ARDS [18] and surgical patients with healthy lungs during the perioperative period [19-21]. Even so, studies on driving pressure and patients with HF are limited. Cross et al. found that, during the graded exercise test, patients with HF had decreased dynamic lung volumes and dynamic lung compliance and increased breath rate, compared to healthy controls [22], suggesting that higher driving pressure is generated during mechanical ventilation. We hypothesized that driving pressure is also associated with outcomes in ventilated patients with $\mathrm{HF}$, although the previous study showed that driving pressure was not associated with in-hospital mortality in non-ARDS patients.

This study mainly assessed the association of driving pressure and mortality in patients with HF and supported the suggestion, using driving pressure limiting strategy in patients with HF during invasive ventilation.

\section{Materials and Methods}

2.1. Database. This retrospective observational cohort study used the Medical Information Mart for Intensive Care-III (MIMIC-III version 1.4) database. The MIMIC-III is a realworld and publicly accessible database containing information on more than 60,000 adult intensive care unit (ICU) stays in Beth Israel Deaconess Medical Center between 2001 and 2012 [23]. The Institutional Review Boards (IRBs) at both the Massachusetts Institute of Technology (MIT) and the Beth Israel Deaconess Medical Center (BIDMC) approved the use of the data for research. The study protocol was approved by the Medical Ethics Committee of the Second Affiliated Hospital of Guangzhou Medical University (2019-ks-11).

2.2. Study Population. Patients with HF were considered eligible for our study. The diagnosis of HF was based on the International Classification of Disease, Ninth Revision (ICD-9) categorized by Quan et al. [24]. As HF may not always be listed as the principal diagnosis, we also included records with HF in any of the first five diagnostic positions according to the diagnostic sequence. The inclusion criteria were as follows: (1) patients were aged $\geq 18$ years; (2) patients received invasive ventilation for at least $48 \mathrm{~h}$; and (3) only the first ICU admission was considered [25].

Patients who were extubated or extirpated during the first $48 \mathrm{~h}$ were excluded. Patients who had incomplete datasets or datasets that did not suffice to calculate driving pressure were also excluded [25].
2.3. Exposure Variable. PEEP and plateau pressure were extracted as the time-weighted values during the first $24 \mathrm{~h}$ of mechanical ventilation and were calculated by averaging the result of the subtraction of PEEP from plateau pressure at each minute during the first $24 \mathrm{~h}$ of ventilation. The minuteby-minute value was obtained by carrying each observation forward until the next observation was recorded [26].

2.4. Covariates. The relevant variables for this study were extracted from the previous report. These variables are commonly used in studies of the relationship between driving pressure and outcomes [14, 26-28]. The following variables were included: demographic characteristics, sequential organ failure assessment (SOFA) score, simplified acute physiology score (SAPS), and Elixhauser comorbidity score. N-terminal pro-B-type natriuretic peptide (NTproBNP) and vital signs (mean arterial pressure (MAP), heart rate, and central venous pressure (CVP)) were recorded $24 \mathrm{~h}$ before and after ICU admission. The laboratory test results from the first day of admission were required [white blood cell (WBC) count, hemoglobin, creatinine, pondus hydrogenii $(\mathrm{PH})$, lowest partial pressure of oxygen in arterial blood $\left(\mathrm{PaO}_{2}\right)$, highest partial pressure of carbon dioxide $\left(\mathrm{PaCO}_{2}\right)$ in arterial blood, and lowest PF ratio]. Some settings and observations needed during mechanical ventilation were considered, including ventilation mode and tidal volume. However, NT-proBNP and CVP were not collected for more than half of the included patients. Dummy variables were used to indicate missing NTproBNP and CVP values $[29,30]$. Body mass index (BMI), calculated as the weight $(\mathrm{kg})$ divided by the square of height (m) [31], and predicted body weight follow the standard formula recommended in the ventilation strategy for patients with ARDS.

2.5. Outcomes. The primary outcome was in-hospital mortality.

2.6. Statistical Analysis. Patient characteristics were analyzed according to the driving pressure tertials. Categorical variables are expressed as numbers and percentages. Continuous variables are expressed as mean and standard deviation (SD) for normal distributions or median and interquartile range (IQR) for skewed distributions. We used the chi-square test, one-way ANOVA, and Kruskal-Wallis test for the comparison of categorical, normally distributed, and nonnormally distributed continuous variables, respectively.

Univariate linear regression analyses and multivariable logistic regression analyses were performed to evaluate the associations between driving pressure and in-hospital mortality. According to the recommendation of the STrengthening the Reporting of OBservational studies in Epidemiology (STROBE) statement [32], analyses were first performed without adjustment. Further analyses cumulatively included adjustment for age, sex (minimally adjusted model), heart rate, MAP, WBC, hemoglobin, creatinine, 
CVP, NT-proBNP, PEEP, $\mathrm{PH}, \mathrm{PaO}_{2}, \mathrm{PaCO}_{2}, \mathrm{PF}$ ratio, Elixhauser score, SOFA score, and SAPS score (fully adjusted model).

We used a generalized additive model (GAM) to identify the nonlinear relationship $[33,34]$. If a nonlinear correlation was observed, a two-piecewise linear regression model was conducted to calculate the threshold effect of the driving pressure on in-hospital mortality in terms of the smoothing plot. When driving pressure and in-hospital mortality were evident in the smoothed curve, the recursive method automatically calculated the threshold, and the maximum model likelihood was used [33]. We performed tests for linear trend by entering the median value of each category of driving pressure as a continuous variable in the models [35].

All analyses were performed using the statistical software package R. version 3.4.3 ( $\mathrm{R}$ Foundation for Statistical Computing, Vienna, Austria) and Free Statistics software version 1.4. $P$ values $<0.05$ (two-sided) were considered statistically significant.

2.7. Sensitivity Analysis. We excluded patients who were ventilated before ICU admission and patients who received ventilation through tracheostomy cannula at any time during the first $48 \mathrm{~h}$ of ventilation as a sensitivity analysis. As a result, patients with chronic obstructive pulmonary disease (COPD) were also excluded because they may have suffered from right $\mathrm{HF}[25,26]$.

\section{Results}

3.1. Participants Selection. Of a total of 61,532 MIMIC-III admissions, 7,221 patients with HF were identified. Figure 1 presents a flowchart of the study. Finally, 632 patients with complete ventilatory data were included.

3.2. Baseline Characteristics. The baseline characteristics are presented in Table 1. Patients enrolled were grouped by the tertials of driving pressure as follows: low group, $\geq 5.00$ to $\leq 12.85 \mathrm{cmH}_{2} \mathrm{O}$; middle group, $\geq 12.86$ to $\leq 16.05 \mathrm{cmH}_{2} \mathrm{O}$; and high group, $\geq 16.10$ to $\leq 35.68 \mathrm{cmH}_{2} \mathrm{O}$. The mean age of all participants was $71.4 \pm 13.5$ years, and $51.11 \%$ were male. Some differences existed between the driving pressure groups with respect to various covariates (BMI, SAPS score, Elixhauser score, CVP, PEEP, driving pressure, hemoglobin, and VT/ PBW). Furthermore, in terms of ventilation mode, the proportions of patients using controlled mechanical ventilation in each group were similar, over one-third, and there was no significant difference between groups $(P=0.996)$.

3.3. Outcomes. The overall in-hospital mortality was $28.64 \%$. The in-hospital mortality of patients with low, middle, and high driving pressure was $27.19 \%, 21.13 \%$, and $38.12 \%$, respectively.

3.4. Driving Pressure and In-Hospital Mortality. The results of the univariate analysis of covariates and in-hospital mortality are shown in Supplementary Table 1. Multivariable logistic regression analyses were used to assess the associations between driving pressure and in-hospital mortality (Table 2). In the minimally adjusted model adjusted for age and sex, driving pressure was positively associated with inhospital mortality (odds ratio [OR], 1.09; 95\% confidence interval [CI], 1.04-1.14; $P=0.0002$ ). Even after adjusting for all potential covariates (Table 2, fully adjusted model), the association remained significant with driving pressure expressed as a continuous variable (OR, 1.12; 95\% CI, 1.06-1.18; $P<0.0001$; Table 2).

When driving pressure entered the fully adjusted model as a categorized variable, the changing trend of the effective value in different driving pressure groups was nonequidistant. The middle and high driving pressure groups had an in-hospital mortality risk that was $26 \%$ lower (OR, 0.74; $95 \%$ CI, $0.44-1.23 ; P=0.242$ ) and $126 \%$ higher (OR, 2.26; $95 \%$ CI, 1.36-3.77; $P=0.002$ ), respectively, compared to low driving pressure patients. $P$ for trend $=0.002$ (Table 2 ).

3.5. Nonlinear Relationship between Driving Pressure and InHospital Mortality. We observed a nonlinear dose-response relationship between driving pressure and in-hospital mortality after adjusting for some covariates (Figure 2). Using a two-piecewise linear regression model, we found that the threshold of driving pressure was $14.27 \mathrm{cmH}_{2} \mathrm{O}$ (Table 3). Above the threshold, the in-hospital mortality rose rapidly (OR, 1.17; 95\% CI, 1.07-1.30; $P<0.001$; Table 3 and Figure 2), and, below the threshold, the estimated doseresponse curve was consistent with a horizontal line $(\mathrm{OR}=0.89 ; 95 \% \mathrm{CI}, 0.75-1.05 ; P=0.176$; Table 3 and Figure 2).

3.6. Sensitivity Analysis. In the sensitivity analysis, we removed 28 patients who received ventilation through tracheostomy cannula at any time during the first $48 \mathrm{~h}$ of ventilation and excluded 171 patients who were ventilated before ICU admission, as well as 47 COPD patients. The association between driving pressure and mortality remained reliable (Supplementary Tables 2-4).

\section{Discussion}

In this retrospective cohort study, driving pressure was independently associated with in-hospital mortality in invasive ventilated patients with $\mathrm{HF}$. We observed a positive association between driving pressure and risk of mortality in patients with $\mathrm{HF}$ who underwent ventilation for more than $48 \mathrm{~h}$. The association was reliable and independent of essential covariates and confounders. To the best of our knowledge, this is the first report of an association between driving pressure and mortality in ventilated patients with HF.

Furthermore, the changing trend of the effective value in different driving pressure groups was nonequidistant, which suggested that the association between driving pressure and in-hospital mortality was likely to be nonlinear. Our study used a generalized additive model and found a nonlinear relationship between driving pressure and mortality in 


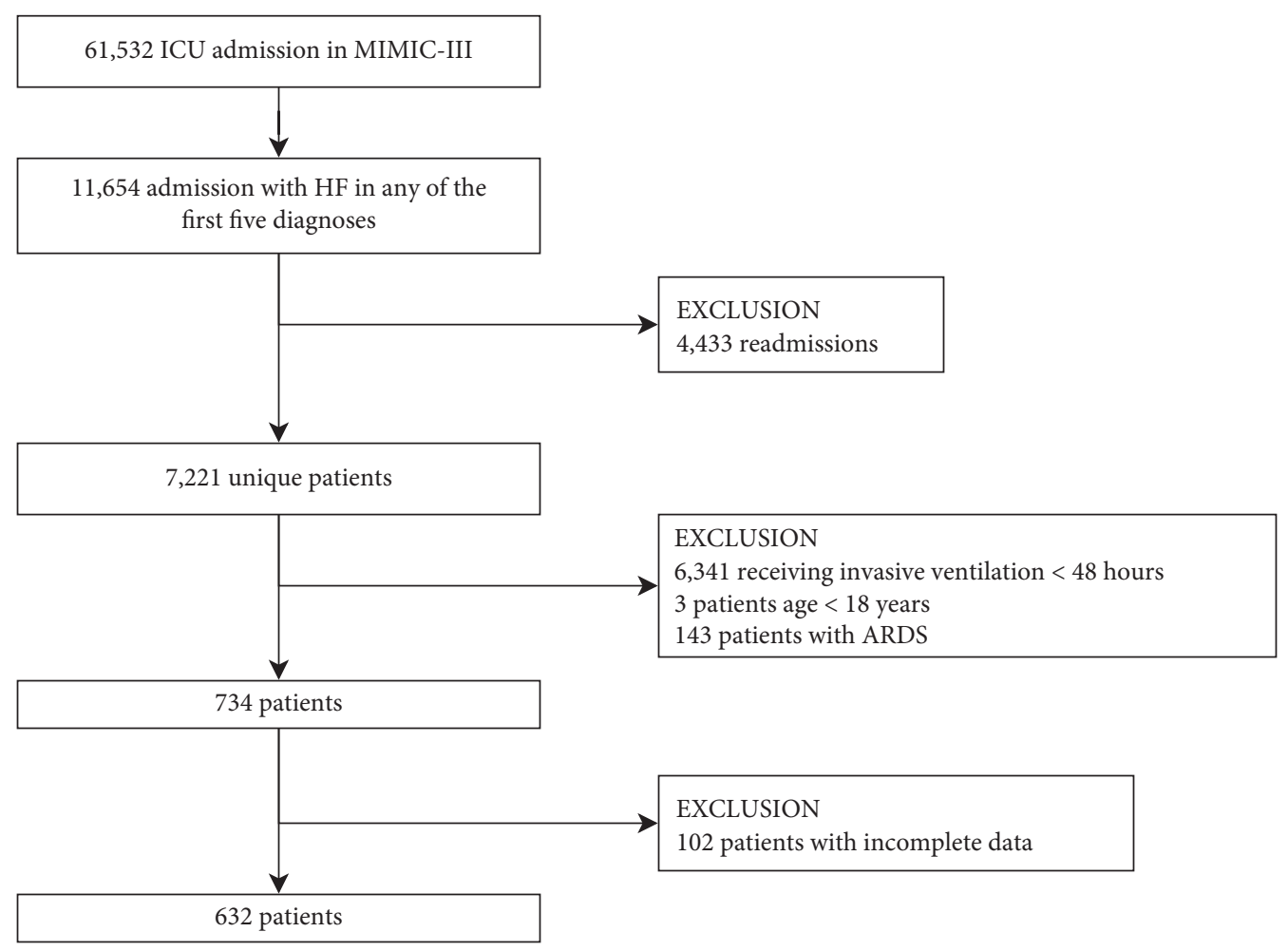

Figure 1: Flowchart of the study cohort.

patients with HF. Nevertheless, it was significantly different that driving pressure affected in-hospital mortality in patients with HF when it was below or above the threshold of $14.27 \mathrm{cmH}_{2} \mathrm{O}$. As assessed at baseline, a positive association was only found for driving pressure above the threshold; otherwise there was no statistical significance, suggesting that a threshold effect was present.

In ICU, low tidal volume ventilation was suggested to avoid ventilator-induced lung injury [36-38]. However, it is important to clarify that ventilator-induced lung injury is not only due to high tidal volume but also due to multiple factors, including airway pressure, PEEP, respiratory rate, and biologic factors [39]. The driving pressure, which is the difference between the plateau pressure and the level of positive end-expiratory pressure, is a good composite indicator. Amato et al. found that driving pressure was the variable that best correlated with survival in patients with ARDS [14]. In Amato's study, each $\mathrm{cmH}_{2} \mathrm{O}$ increment of driving pressure was associated with a hazard ratio (HR) of 1.049 in terms of the risk of death in patients with ARDS [14], which was similar to the result reported by Guérin et al. [27]. In our cohort, with each $\mathrm{cmH}_{2} \mathrm{O}$ increment, driving pressure was associated with an $\mathrm{OR}$ of 1.12 in the risk of inhospital mortality in patients with HF. This may be due to the pulmonary edema which manifests in patients with HF and ARDS despite their different mechanisms [22]. Lung compliance has been found to be significantly reduced when patients suffer from pulmonary edema, while higher driving pressure promotes lung overstress, leading to lung injury [16]. Therefore, it is reasonable that driving pressure may affect the mortality of patients with HF in the same manner as in patients with ARDS. Nevertheless, it is worth noting that the relationship may be biased in obese patients because of the unique mechanics of the respiratory system (lower total lung capacity and functional residual capacity and higher pleural pressure and airway resistance) [40]. The baseline characteristics of the current patients indicated that the BMI was higher in the high driving pressure cohort than in the other two groups, which can be interpreted as the stiffness of the chest-wall rather than the worsening in the lung elastance [41].

This study is the first to report a nonlinear relationship between driving pressure and mortality. We calculated a threshold of approximately $14 \mathrm{cmH}_{2} \mathrm{O}$, while the effect below and above the threshold was completely different. This threshold was similar to the cutoff values reported in several previous studies focused on outcomes of patients with ARDS. The cutoff values of driving pressure were $13 \mathrm{cmH}_{2} \mathrm{O}$ [27], $14 \mathrm{cmH}_{2} \mathrm{O}$ [42], and $15 \mathrm{cmH}_{2} \mathrm{O}$ [14], respectively. A recent prospective longitudinal cohort study of patients with ARDS showed that the lung function and lung fibrosis index at 6 months were worse in high driving pressure group $(\geq 13$ $\left.\mathrm{cmH}_{2} \mathrm{O}\right)$ than in the low driving pressure group $(<13$ $\mathrm{cmH}_{2} \mathrm{O}$ ) [43]. Based on the results of our study, we inferred that the threshold could explain why researchers chose driving pressure around $14 \mathrm{cmH}_{2} \mathrm{O}$ as a cutoff value.

However, Schmidt et al. showed that the driving pressure was not associated with hospital mortality in non-ARDS patients (include HF patients) using the MIMIC-II database for analyses [26]. In Schmidt's research, only 9.16\% (57/622) of patients were diagnosed with cardiac-related diseases (myocardial infarction, acute coronary syndrome, and 
TABLE 1: Baseline characteristics of participants.

\begin{tabular}{|c|c|c|c|c|}
\hline \multirow[b]{2}{*}{ Covariate } & \multicolumn{3}{|c|}{ Driving pressure } & \multirow[b]{2}{*}{$P$ value } \\
\hline & $\begin{array}{c}\text { Low }(5.00-12.85) \\
n=217\end{array}$ & $\begin{array}{c}\text { Mid }(12.86-16.05) \\
n=213\end{array}$ & $\begin{array}{c}\text { High }\left(\begin{array}{l}(16.10-35.68) \\
n=202\end{array}\right. \\
\end{array}$ & \\
\hline \multicolumn{5}{|l|}{ Baseline characteristics } \\
\hline Age (years) & $71.83 \pm 14.55$ & $72.20 \pm 12.96$ & $70.03 \pm 12.76$ & 0.216 \\
\hline Sex (male) & $121(55.76 \%)$ & $110(51.64 \%)$ & $92(45.54 \%)$ & 0.11 \\
\hline BMI $\left(\mathrm{kg} / \mathrm{m}^{2}\right)$ & $27.9 \pm 8.0$ & $29.7 \pm 7.9$ & $32.5 \pm 9.7$ & $<0.001$ \\
\hline PBW (kg) & $62.9 \pm 11.2$ & $62.2 \pm 10.5$ & $60.9 \pm 11.1$ & 0.163 \\
\hline \multicolumn{5}{|l|}{ Disease severity score } \\
\hline SAPS & $21.73 \pm 4.27$ & $22.43 \pm 4.31$ & $22.95 \pm 4.58$ & 0.017 \\
\hline SOFA & $6.27 \pm 3.27$ & $6.48 \pm 3.24$ & $6.79 \pm 3.14$ & 0.18 \\
\hline Elixhauser index & $11.00(6.00-15.00)$ & $8.00(3.00-13.00)$ & $10.00(6.00-15.00)$ & 0.01 \\
\hline \multicolumn{5}{|l|}{ Vital signs } \\
\hline Heart rate $(\mathrm{bpm})$ & $85.03 \pm 16.42$ & $84.97 \pm 16.40$ & $86.85 \pm 16.71$ & 0.421 \\
\hline Respiratory rate (bpm) & $18.74 \pm 3.90$ & $19.03 \pm 3.80$ & $18.97 \pm 4.30$ & 0.727 \\
\hline MAP $(\mathrm{mmHg})$ & $77.59 \pm 11.35$ & $75.47 \pm 10.23$ & $76.92 \pm 9.56$ & 0.099 \\
\hline $\mathrm{CVP}\left(\mathrm{cmH}_{2} \mathrm{O}\right)$ & $15.00(12.00-19.50)$ & $17.00(14.00-23.00)$ & $20.00(16.00-26.00)$ & $<0.001$ \\
\hline \multicolumn{5}{|l|}{ Laboratory examination } \\
\hline $\mathrm{WBC}\left(\times 10^{9} / \mathrm{L}\right)$ & $14.99 \pm 7.23$ & $15.58 \pm 7.31$ & $16.31 \pm 16.92$ & 0.856 \\
\hline Hemoglobin $(\mathrm{g} / \mathrm{L})$ & $12.26 \pm 2.06$ & $11.83 \pm 2.05$ & $11.68 \pm 1.90$ & 0.01 \\
\hline NT-proBNP (pg/ml) & $5016.00(1341.50-15748.25)$ & $3753.00(1344.50-6903.50)$ & $4293.50(2142.25-17458.50)$ & 0.425 \\
\hline Creatinine $(\mathrm{mg} / \mathrm{ml})$ & $1.53 \pm 1.82$ & $1.41 \pm 1.24$ & $1.46 \pm 1.07$ & 0.626 \\
\hline $\mathrm{PH}$ & $7.29 \pm 0.11$ & $7.29 \pm 0.11$ & $7.28 \pm 0.10$ & 0.802 \\
\hline $\mathrm{PO}_{2}(\mathrm{mmHg})$ & $79.00(63.00-115.00)$ & $80.00(66.00-104.00)$ & $76.50(61.75-99.00)$ & 0.239 \\
\hline $\mathrm{PCO}_{2}(\mathrm{mmHg})$ & $53.41 \pm 20.43$ & $54.11 \pm 20.48$ & $57.65 \pm 20.33$ & 0.081 \\
\hline $\mathrm{PF}$ ratio & $166.67(108.54-245.83)$ & $153.25(106.87-240.00)$ & $156.33(106.79-218.78)$ & 0.67 \\
\hline \multicolumn{5}{|c|}{ Parameters of mechanical ventilation } \\
\hline $\operatorname{PEEP}\left(\mathrm{cmH}_{2} \mathrm{O}\right)$ & $7.36 \pm 3.16$ & $7.22 \pm 3.21$ & $6.64 \pm 2.78$ & 0.04 \\
\hline VT/PBW & $8.29 \pm 1.86$ & $8.77 \pm 1.66$ & $9.22 \pm 2.35$ & $<0.001$ \\
\hline $\mathrm{CMV}$ & $77(36.5 \%)$ & $75(35.7 \%)$ & $74(35.1 \%)$ & 0.996 \\
\hline \multicolumn{5}{|l|}{ Outcome } \\
\hline In-hospital mortality & $59(27.19 \%)$ & $45(21.13 \%)$ & $77(38.12 \%)$ & $<0.001$ \\
\hline
\end{tabular}

TABLE 2: Multivariable logistic regression analyses of driving pressure and in-hospital mortality.

\begin{tabular}{lccccc}
\hline Variable & $\begin{array}{c}\text { Crude model, } \\
n=632\end{array}$ & $P$ value & $\begin{array}{c}\text { Minimally adjusted model, } \\
n=632\end{array}$ & $P$ value & $\begin{array}{c}\text { Fully adjusted model, } \\
n=603\end{array}$ \\
\hline $\begin{array}{l}\text { Driving pressure } \\
\left(\mathrm{cmH}_{2} \mathrm{O}\right)\end{array}$ & $1.07(1.02,1.11)$ & 0.0022 & $1.09(1.04,1.14)$ & 0.0002 & $1.12(1.06,1.18)$ \\
\hline Driving pressure tertials & Reference & & & $<0.001$ \\
Low & $0.72(0.46,1.12)$ & 0.1430 & $0.71(0.45,1.12)$ & 0.1376 & $0.74(0.44,1.23)$ \\
Mid & $1.65(1.09,2.49)$ & 0.0174 & $1.89(1.23,2.90)$ & 0.0039 & $2.26(1.36,3.77)$ \\
High & 0.009 & 0.0019 & 0.002 & 0.242 \\
$P$ for trend & & & & 0.002 \\
\hline
\end{tabular}

Crude model: no other covariates were adjusted. Minimally adjusted model: we adjusted age and sex. Fully adjusted model: we adjusted age, sex, BMI, heart rate, MAP, WBC, hemoglobin, creatinine, CVP, NT-proBNP, PEEP, $\mathrm{PH}, \mathrm{PaO}_{2}, \mathrm{PaCO}_{2}$, PF ratio, ventilation mode (CMV), VT/PBW, Elixhauser score, SOFA score, and SAPS score.

congestive HF). Therefore, their results do not represent the association between driving pressure and mortality in patients with HF. Moreover, they excluded patients who were transferred from other hospitals to eliminate patients ventilated before ICU admission [26], which may have changed the result. In sensitivity analysis, we also excluded 171 patients who were ventilated before ICU admission and the result remained stable. Besides, the MIMIC-III database is an extension of the MIMIC-II database; it incorporates the data contained in the old version (collected between 2001 and 2008) and augments it with newly collected data between 2008 and 2012. Our study included 632 patients with 


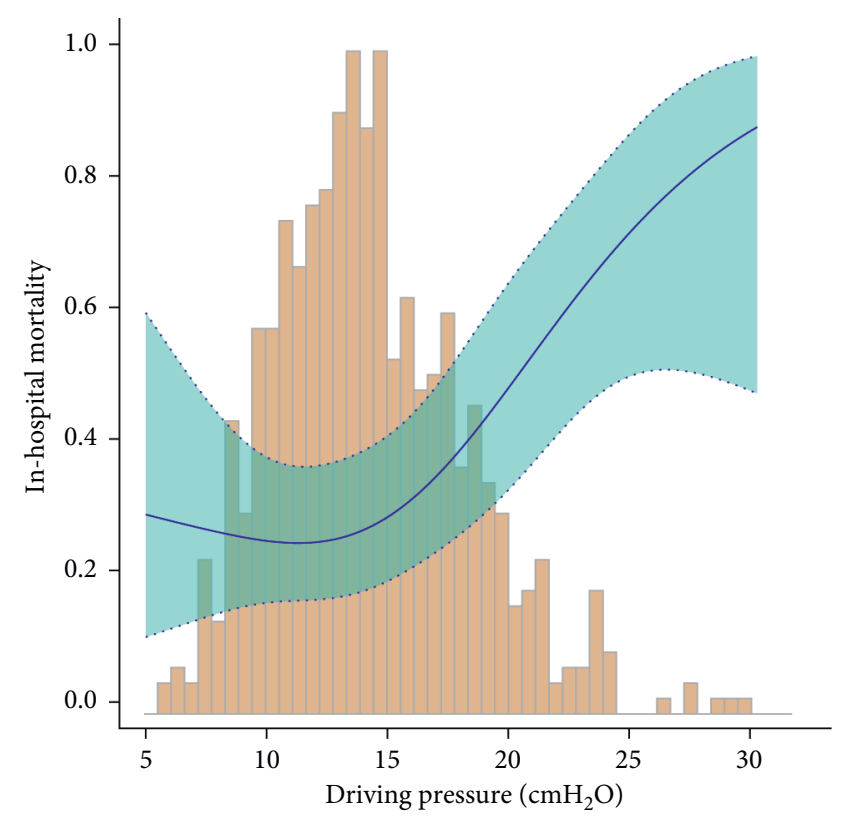

FIgURE 2: Nonlinear dose-response relationship between driving pressure and in-hospital mortality. Adjustment factors included age, sex, $\mathrm{BMI}$, heart rate, MAP, WBC, hemoglobin, creatinine, CVP, NT-proBNP, PEEP, $\mathrm{PH}, \mathrm{PaO}_{2}, \mathrm{PaCO}_{2}$, $\mathrm{PF}$ ratio, ventilation mode (CMV), VT/PBW, Elixhauser score, SOFA score, and SAPS score. The blue line and green area represent the estimated values and their corresponding $95 \%$ confidence intervals, respectively.

TABLE 3: Threshold effect analysis of driving pressure on in-hospital mortality.

\begin{tabular}{lccc}
\hline Threshold of driving pressure & OR & $95 \% \mathrm{CI}$ & $P$ value \\
\hline$<14.27 \mathrm{cmH}_{2} \mathrm{O}$ & 0.89 & $0.75-1.05$ & 0.176 \\
$\geq 14.27 \mathrm{cmH}_{2} \mathrm{O}$ & 1.17 & $1.07-1.30$ & $<0.001$ \\
\hline
\end{tabular}

Adjustment factors included age, sex, BMI, heart rate, MAP, WBC, hemoglobin, creatinine, CVP, NT-proBNP, PEEP, $\mathrm{PH}, \mathrm{PaO}_{2}, \mathrm{PaCO}_{2}$, PF ratio, ventilation mode (CMV), VT/PBW, Elixhauser score, SOFA score, and SAPS score.

HF whose data were analyzed and adjusted for 20 potential confounding factors. The relationship between driving pressure and mortality in patients with HF was stable.

In our study, we followed Serpa Neto et al. 's method to include and exclude patients [25]. However, as there was no evidence to show that being ventilated through a tracheostomy cannula had any effect on driving pressure, we did not exclude these patients. In sensitivity analysis, no different effect was found after excluding the 28 patients who were ventilated through a tracheostomy cannula within $48 \mathrm{~h}$ of ICU admission.

There are several limitations to this study. First, conclusions can be generalized to the patients with HF who were ventilated for more than $48 \mathrm{~h}$ only. Second, as in all observational studies, there may have been some uncontrolled potential confounders. Besides, considering that the MIMIC-III database did not contain the diagnosis of right HF and the lack of patients diagnosed with cor pulmonale, it is impossible to exclude the right HF from our study. In the sensitivity analyses, we eliminated 47 patients with COPD who may have suffered undiagnosed cor pulmonale, and the association was still stable. Third, in terms of ventilation mode, a recent study conducted by Di Mussi et al. showed that, during pressure support ventilation (PSV), the increase in neuroventilatory drive could lead to the variations in respiratory drive and effort, which may have an impact on driving pressure [44]. In fact, we did not strictly limit to volume control ventilation, and some patients in our study received assisted ventilation mode, which imparted a certain bias to the results. However, there was no significant difference in ventilation patterns among groups at baseline and after adjusting for ventilation mode in multivariate analysis, and our results remained robust. In addition, due to the limitations of the database, there was no explanation about how the plateau pressure (Pplat) was measured and recorded. This should be carefully considered in the next step of our real-world research. Future studies will focus on random control trial researches to confirm the causal relationship between driving pressure and mortality.

\section{Conclusion}

There was a nonlinear relationship between driving pressure and mortality in patients with HF who were ventilated for more than $48 \mathrm{~h}$, and this relationship was associated with increased in-hospital mortality when the driving pressure was more than $14.27 \mathrm{cmH}_{2} \mathrm{O}$.

\section{Abbreviations}

ARDS: $\quad$ Acute respiratory distress syndrome

bpm: $\quad$ Beats per minute

BMI: $\quad$ Body mass index

CVP:

CMV:

HF:

ICU:

MAP:

Central venous pressure

Control mechanical ventilation

Heart failure

MIMIC-

Intensive care unit

III:

NT-

proBNP:

$\mathrm{PaCO}_{2}$ : Partial pressure of carbon dioxide in arterial blood

$\mathrm{PaO}_{2}: \quad$ Partial pressure of oxygen in arterial blood

PBW: $\quad$ Predicted body weight

PF ratio: $\quad \mathrm{PaO}_{2} / \mathrm{FiO}_{2}$ ratio

PEEP: $\quad$ Positive end-expiratory pressure

SAPS Simplified acute physiology score

score:

SOFA Sequential organ failure assessment score

score:

VT/PBW: Tidal volume/predicted body weight.

\section{Data Availability}

All data in the article can be obtained from the MIMIC-III database (https://mimic.physionet.org/). 


\section{Ethical Approval}

The study protocol was approved by the Medical Ethics Committee of the Second Affiliated Hospital of Guangzhou Medical University (2019-ks-11).

\section{Conflicts of Interest}

The authors declare that they have no conflicts of interest.

\section{Authors' Contributions}

Qilin Yang conducted data analysis and wrote the manuscript. Jiezhao Zheng modified the manuscript and interpreted the analysis. Xiaohua Chen conducted the data collection. Weiyan Chen conducted data analysis and reviewed the manuscript. Deliang Wen conducted data collection and data interpretation. Xuming Xiong and Zhenhui Zhang designed the study and conducted data analysis and reviewed the manuscript. The authors Qilin Yang and Jiezhao Zheng contributed equally to this study.

\section{Acknowledgments}

The authors wish to thank the team of the Laboratory for Computational Physiology from the Massachusetts Institute of Technology (LCP-MIT) for keeping the MIMIC-III databases available.

\section{Supplementary Materials}

Supplementary Materials: additional DOC file includes supplementary tables showing the results of the sensitivity analyses. (Supplementary Materials)

\section{References}

[1] M. Metra and J. R. Teerlink, "Heart failure," The Lancet, vol. 390, no. 10106, pp. 1981-1995, 2017.

[2] A. L. Bui, T. B. Horwich, and G. C. Fonarow, "Epidemiology and risk profile of heart failure," Nature Reviews Cardiology, vol. 8, no. 1, pp. 30-41, 2011.

[3] E. J. Benjamin, P Muntner, A Alonso et al., "Heart disease and stroke statistics-2019 update: a report from the American heart association," Circulation, vol. 139, no. 10, pp. e56-e528, 2019.

[4] L. H. Curtis, D. J. Whellan, B. G. Hammill et al., "Incidence and prevalence of heart failure in elderly persons, 1994-2003," Archives of Internal Medicine, vol. 168, no. 4, pp. 418-424, 2008.

[5] L. R. Loehr, W. D. Rosamond, P. P. Chang, A. R. Folsom, and L. E. Chambless, "Heart failure incidence and survival (from the Atherosclerosis Risk in Communities study)," The American Journal of Cardiology, vol. 101, no. 7, pp. 1016-1022, 2008.

[6] W. Jonathan, M. Ornstein, A. R. Tonelli, V. Menon, and R. W. Ashton, "State of the evidence: mechanical ventilation with PEEP in patients with cardiogenic shock," Heart, vol. 99, no. 24, pp. 1812-1817, 2013.

[7] M. Hongisto, J. Lassus, T. Tarvasmaki et al., "Use of noninvasive and invasive mechanical ventilation in cardiogenic shock: a prospective multicenter study," International Journal of Cardiology, vol. 230, pp. 191-197, 2017.

[8] S. Vallabhajosyula, K. Kashani, S. M. Dunlay et al., "Acute respiratory failure and mechanical ventilation in cardiogenic shock complicating acute myocardial infarction in the USA, 2000-2014," Annals of Intensive Care, vol. 9, no. 1, 2019.

[9] J. J. Marini, P. R. M. Rocco, and L. Gattinoni, "Static and dynamic contributors to ventilator-induced lung injury in clinical practice. Pressure, energy, and power," American Journal of Respiratory and Critical Care Medicine, vol. 201, no. 7, pp. 767-774, 2020.

[10] A. Fogagnolo, S. Grasso, M. Dres et al., "Focus on renal blood flow in mechanically ventilated patients with SARS-CoV-2: a prospective pilot study," Journal of Clinical Monitoring and Computing, pp. 1-7, 2021.

[11] M. R. Wilson and M. Takata, "Inflammatory mechanisms of ventilator-induced lung injury: a time to stop and think?" Anaesthesia, vol. 68, no. 2, pp. 175-178, 2013.

[12] T. Tonetti, F. Vasques, F. Rapetti et al., "Driving pressure and mechanical power: new targets for VILI prevention," Annals of Translational Medicine, vol. 5, no. 14, p. 286, 2017.

[13] S. Saffaran, A Das, J. G Laffey, J. G Hardman, N Yehya, and D. G Bates, "Utility of driving pressure and mechanical power to guide protective ventilator settings in two cohorts of adult and pediatric patients with acute respiratory distress syndrome: a computational investigation," Critical Care Medicine, vol. 48, no. 7, pp. 1001-1008, 2020.

[14] M. B. P. Amato, M. O. Meade, A. S. Slutsky et al., "Driving pressure and survival in the acute respiratory distress syndrome," New England Journal of Medicine, vol. 372, no. 8, pp. 747-755, 2015.

[15] H. Aoyama, T. Pettenuzzo, K. Aoyama, R. Pinto, M. Englesakis, and E. Fan, "Association of driving pressure with mortality among ventilated patients with acute respiratory distress syndrome," Critical Care Medicine, vol. 46, no. 2, pp. 300-306, 2018.

[16] D. Chiumello, E. Carlesso, M. Brioni, and M. Cressoni, "Airway driving pressure and lung stress in ARDS patients," Critical Care, vol. 20, no. 1, p. 276, 2016.

[17] S. Grasso, L. Mirabella, F. Murgolo et al., "Effects of positive end-expiratory pressure in "high compliance" severe acute respiratory syndrome coronavirus 2 acute respiratory distress syndrome*," Critical Care Medicine, vol. 48, no. 12, pp. e1332-e1336, 2020.

[18] H. P. Wu, H. C Hu, C. M Chu, and K. C Kao, "The association between higher driving pressure and higher mortality in patients with pneumonia without acute respiratory distress syndrome," Journal of the Formosan Medical Association = Taiwan yizhi, vol. 120, no. 1 Pt 1, pp. 204-211, 2021.

[19] P. L. Silva, D. Negrini, and P. R. Macêdo Rocco, "Mechanisms of ventilator-induced lung injury in healthy lungs," Best Practice \& Research Clinical Anaesthesiology, vol. 29, no. 3, pp. 301-313, 2015.

[20] A. S. Neto, S. N. T. Hemmes, C. S. V. Barbas et al., "Association between driving pressure and development of postoperative pulmonary complications in patients undergoing mechanical ventilation for general anaesthesia: a meta-analysis of individual patient data," The Lancet Respiratory Medicine, vol. 4, no. 4, pp. 272-280, 2016.

[21] S. Spadaro, S. Grasso, D. S. Karbing et al., "Physiological effects of two driving pressure-based methods to set positive end-expiratory pressure during one lung ventilation," Journal of Clinical Monitoring and Computing, vol. 35, no. 5, pp. 1149-1157, 2021. 
[22] T. J. Cross, S. Sabapathy, K. C. Beck, N. R. Morris, and B. D. Johnson, "The resistive and elastic work of breathing during exercise in patients with chronic heart failure," $E u-$ ropean Respiratory Journal, vol. 39, no. 6, pp. 1449-1457, 2012.

[23] A. E. W. Johnson, T. J. Pollard, L. Shen et al., "MIMIC-III, a freely accessible critical care database," Scientific Data, vol. 3, no. 1, Article ID 160035, 2016.

[24] H. Quan, G. A. Parsons, and W. A. Ghali, "Validity of procedure codes in International Classification of Diseases, 9th revision, clinical modification administrative data," Medical Care, vol. 42, no. 8, pp. 801-809, 2004.

[25] A. Serpa Neto, R. O. Deliberato, A. E. W. Johnson et al., "Mechanical power of ventilation is associated with mortality in critically ill patients: an analysis of patients in two observational cohorts," Intensive Care Medicine, vol. 44, no. 11, pp. 1914-1922, 2018.

[26] M. Schmidt, A. C. K. B. Amaral, E. Fan, and G. D. Rubenfeld, "Driving pressure and hospital mortality in patients without ARDS," Chest, vol. 153, no. 1, pp. 46-54, 2018.

[27] C. Guérin, L. Papazian, G. Reignier, L. Ayzac, A. Loundou, and J. M. Forel, "Effect of driving pressure on mortality in ARDS patients during lung protective mechanical ventilation in two randomized controlled trials," Critical Care, vol. 20, no. $1,2016$.

[28] A. De Jong, J. Cossic, D. Verzilli et al., "Impact of the driving pressure on mortality in obese and non-obese ARDS patients: a retrospective study of 362 cases," Intensive Care Medicine, vol. 44, no. 7, pp. 1106-1114, 2018.

[29] M. Feng, J. I. McSparron, D. T. Kien et al., "Transthoracic echocardiography and mortality in sepsis: analysis of the MIMIC-III database," Intensive Care Medicine, vol. 44, no. 6, pp. 884-892, 2018.

[30] C. Vetter, E. E. Devore, L. R. Wegrzyn et al., "Association between rotating night shift work and risk of coronary heart disease among women," Journal of the American Medical Association, vol. 315, no. 16, p. 1726, 2016.

[31] K. B. Smith and M. S. Smith, "Obesity Statistics," Prim Care, vol. 43, no. 1, pp. 121-135, 2016.

[32] E. Von Elm, "Strengthening the Reporting of Observational Studies in Epidemiology (STROBE) statement: guidelines for reporting observational studies," BMJ, vol. 335, no. 7624, pp. 806-808, 2007.

[33] X. Yu, "Threshold effects of moderately excessive fluoride exposure on children's health: a potential association between dental fluorosis and loss of excellent intelligence," Environment International, vol. 118, pp. 116-124, 2018.

[34] X. Kong, "Platelet count affects efficacy of folic acid in preventing first stroke," Journal of the American College of Cardiology, vol. 71, no. 19, pp. 2136-2146, 2018.

[35] S. Y. Park, N. D. Freedman, C. A. Haiman, L. L. Marchand, L. R. Wilkens, and V. W. Setiawan, "Association of coffee consumption with total and cause-specific mortality among nonwhite populations," Annals of Internal Medicine, vol. 167, no. 4, pp. 228-235, 2017.

[36] R. G. Brower, "Ventilation with lower tidal volumes as compared with traditional tidal volumes for acute lung injury and the acute respiratory distress syndrome," New England Journal of Medicine, vol. 342, no. 18, pp. 1301-1308, 2000.

[37] V. M. Ranieri, "Acute respiratory distress syndrome: the Berlin Definition," Journal of the American Medical Association, vol. 307, no. 23, pp. 2526-2533, 2012.

[38] R. P. Dellinger, "Surviving Sepsis Campaign: international guidelines for management of severe sepsis and septic shock," Intensive Care Medicine, vol. 39, no. 2, pp. 165-228, 2012.
[39] A. S. Slutsky and V. M. Ranieri, "Ventilator-induced lung injury," New England Journal of Medicine, vol. 369, no. 22, pp. 2126-2136, 2013.

[40] K. Hibbert, M. Rice, and A. Malhotra, "Obesity and ARDS," Chest, vol. 142, no. 3, pp. 785-790, 2012.

[41] A. De Jong, H. Wrigge, G. Hedenstierna et al., "How to ventilate obese patients in the ICU," Intensive Care Medicine, vol. 46, no. 12, pp. 2423-2435, 2020.

[42] G. Bellani, J. G. Laffey, and T. Pham, "Epidemiology, patterns of care, and mortality for patients with acute respiratory distress syndrome in intensive care units in 50 countries," Journal of the American Medical Association, vol. 315, no. 8, pp. 788-800, 2016.

[43] J. C. Toufen, S. R. R. De Santis, A. S. Hirota et al., "Driving pressure and long-term outcomes in moderate/severe acute respiratory distress syndrome," Annals of Intensive Care, vol. 8, no. 1, p. 119, 2018.

[44] R. Di Mussi, S. Spadaro, C. A. Volta et al., "Continuous assessment of neuro-ventilatory drive during $12 \mathrm{~h}$ of pressure support ventilation in critically ill patients," Critical Care (London, England), vol. 24, no. 1, p. 652, 2020. 